\title{
Presynaptic Congenital Myasthenic Syndrome 6
}

National Cancer Institute

\section{Source}

National Cancer Institute. Presynaptic Congenital Myasthenic Syndrome 6. NCI

Thesaurus. Code C132292.

Congenital myasthenic syndrome caused by mutation(s) in the CHAT gene, encoding choline O-acetyltransferase. It is inherited in an autosomal recessive manner. 